\title{
INTERVAL TYPE-2 FUZZY BASED NEURAL NETWORK FOR HIGH RESOLUTION REMOTE SENSING IMAGE SEGMENTATION
}

\author{
Wang Chunyan ${ }^{1}$ Xu Aigong ${ }^{2}$ Li Chao $^{3}$ Zhao Xuemei $^{2}$ \\ 1. School of Mining Industry and Technology, Liaoning Technical University Huludao 125105; 2. School of Geomatics, Liaoning \\ Technical University, Fuxin 123000; 3 Yunnan Technology Center of Basic Surveying and Mapping, Yunnan 650034 \\ China-(wcy0211@126.com; china xu_ag@126.com; 719913163@qq.com; 374010101@qq.com)
}

\section{CommissionVII, WG VII/4}

\begin{abstract}
KEY WORDS: Interval Type-2 Fuzzy Model, High Resolution Remote Sensing Image, Footprint of Uncertainty, Image Segmentation, Neuron Networks
\end{abstract}

\begin{abstract}
:
Recently, high resolution remote sensing image segmentation is a hot issue in image processing procedures. However, it is a difficult task. The difficulties derive from the uncertainties of pixel segmentation and decision-making model. To this end, we take spatial relationship into consideration when constructing the interval type-2 fuzzy neural networks for high resolution remote sensing image segmentation. First, the proposed algorithm constructs a Gaussian model as a type- 1 fuzzy model to describe the uncertainty contained in the image. Second, the interval type- 2 fuzzy model is obtained by blurring the mean and variance in type-1 model. The proposed interval type- 2 model can strengthen the expression of uncertainty and simultaneously decrease the uncertainty in the decision model. Then the fuzzy membership function itself and its upper and lower fuzzy membership functions of the training samples are used as the input of the neuron network which acts as the decision model in the proposed algorithm. Finally, the relationship of neighbour pixels is taken into consideration and the fuzzy membership functions of the detected pixel and its neighbourhood are used to decide the class of each pixel to get the final segmentation result. The proposed algorithm, FCM and HMRF-FCM algorithm and an interval type-2 fuzzy neuron networks without spatial relationships are performed on synthetic and real high resolution remote sensing images. The qualitative and quantitative analyses demonstrate the efficient of the proposed algorithm, especially for homogeneous regions which contains a great difference in its gray level (for example forest).
\end{abstract}

\section{INTRODUCTION}

Image segmentation is an important task in remote sensing image processing. High-resolution remote sensing data can present more clearly detail information of ground objects, which greatly eliminates the uncertainty of pixel feature caused by mixed pixel and has great potential and advantages in accurate segmentation of ground objects. However, high resolution also brings some new problems and difficulties for remote sensing image segmentation. For example, (1) in more accurate spatial scale, the variability of pixel spectrum measure within a ground object in remote sensing image increases. As a consequence, ground objects with the same type will present more heterogeneity and spatial fragmentation (e.g., the asymmetric and multimodal distributions of the same ground object)(Bruzzone and Carlin., 2006); the similarity enhancement of different ground objects (e.g., the increase of overlapping region of the pixel spectrum measure distribution curves of different ground objects) can increase the uncertainty of pixel generic attribute; (2) Because the detailed ground information covered by high-resolution remote sensing image is rich, the complexity and diversify of ground objects and the lack of real ground coverage information further increase the uncertainty of segmentation decision-making. The above two uncertainties bring new problems and difficulties for the segmentation of high-resolution remote sensing image.

At present, the fuzzy clustering method is one of the most commonly used and effective methods to solve the problem of uncertain data clustering. The Fuzzy C-Means (FCM) (Bezdek., 1981) algorithm, incorporated with the correlation of pixels, is the most widely used method for its capacity to effectively deal with the pixel spatial correlation and fully describe the fuzziness and uncertainty of spectral measure in the image and other excellent features. The algorithm uses membership function to represent the pixel uncertainty, build the object function by defining the non-similarity measure, define the deterministic function model neighborhood relationship of the neighborhood pixel spectrum measure (Cai et al., 2007; Ahmed et al., 2002) or build the correlation model (Chatzisand and Varvarigon., 2008; Liu. et al., 2007) of the neighborhood pixels by Markov Random Field (MRF), and obtain the optimum fuzzy segmentation by solving the object function. Although these algorithms can smooth noise to a certain extent, effectively solve the problem of pixel uncertainty caused by the spatial correlation of pixels, and thus improve the segmentation accuracy of the algorithms, they cannot deal with the influence of uncertainty of decision-making on the segmentation results in high-resolution image segmentation.

In recent years, type-2 fuzzy theory (Karnik and Mendel., 2001; Jonr et al., 2000; Liang and Mendel .,2001; Mendel., 2000), applied to deal with the uncertainty problems, is a new method. Based on this theory, the image model with the primary membership function and secondary membership function are built, which can effectively deal with the above two kinds of uncertainty. However, Type-2 fuzzy theory needs to define the secondary membership function based on

\footnotetext{
* Xu Aigong Professor in Liaoning Technical University. His research interests major in the integration of GNSS, GIS and ITS and their application. E-mail: xu_ag@126.com
} 
the primary membership, and the definition of the secondary membership function will have a decisive influence on the uncertainty processing. In fact, the most commonly used theory is the data modeling based on interval type-2 fuzzy theory, which defines the secondary membership as the interval function value as one. It has been applied to the time-sequence decision (Karnik et al., 1999), speech recognition (Melin et al., 2006), noise processing (Castillo and Melin., 2004), etc. The type-reduction problem (the type- 2 fuzzy model reduced to type- 1 fuzzy model, namely build the decision-making model) has been the focus of the type-2 fuzzy theory, and the quality of this decision-making model can directly affect the recognition accuracy. To build the neural network ( Sanchez and Melin., 2010; Juang and Tsao., 2008; Lin et al., 2014; Ahmadieh et al., 2011) with the information provided by the interval type- 2 fuzzy model is the most commonly used method with the theory modeling decision-making model, which connect all the information provided by the interval type- 2 fuzzy model in the form of weighted sum, determine whether the information play a role in the objective function with the weight symbol (positive weight means the membership function is activated, negative and zero weight means the membership function is restrained), and judge the importance of the weight with the value of weight (the greater the weight is, the more important its role will be). Compared with the traditional modeling method, this modeling method can greatly improve the quality of decision-making model. But the above segmentation method doesn't consider

that the influence of spatial correlation of pixels on segmentation accuracy. Thus, it is sensitive to noise.

To this end, this paper proposes a high-resolution remote sensing image supervised segmentation method based on interval type-2 fuzzy and neural network integrated with spatial relationship. First of all, in order to strengthen the generic uncertainty representation and increase the segmentation decision-making information, the membership function model with supervised sampling is built to process the pixel generic uncertainty in high-resolution image, and then the interval type- 2 fuzzy model by fuzzing the mean or standard deviation in the type-1 fuzzy model is built. Then the fuzzy neural network model is built by taking the original membership and upper and lower membership of the training sample in all types as input. And finally implement the segmentation decision-making by integrating the spatial relationship.

\section{DESCRIPTION OF THE PROPOSED ALGORITHM}

\subsection{Fuzzy image model}

(1) Type-1 fuzzy image model

For the given image $\boldsymbol{Z}=\left\{z_{j}, j=1, \ldots, n\right\}$, where $j$ is the index of pixel, $n$ is the total number of pixels, $z_{j}$ is the spectrum measure of $j$ th pixel. The fuzzy set $\left\{\boldsymbol{U}_{n \times m} \mid u_{j i} \in[0,1]\right\}$ is built, where $U$ is matrix with $m \times n, m$ refers to the type, $i=1, \ldots, m$ is the type index, $u_{j i}$ is matrix element, which stands for the membership of $j$ th pixel to the $i$ th type, where the value region is $0 \leq u_{j i} \leq 1$ and meet the constraint condition $\sum_{j=1}^{n} u_{j i}=1$. In this paper, Gaussian Function Model ${ }^{[19]}$, a representative general distribution model with good calculated performance, is used as the membership function model of the image (Type-1 fuzzy image model),

$$
u_{j i}=\beta_{i} \times \exp \left\{-\frac{\left(z_{j}-\mu_{i}\right)^{2}}{2 \sigma_{i}^{2}}\right\}
$$

where $u_{j i}$ is the membership of $j$ th pixel to the $i$ th type, coefficient $0 \leq \beta_{i} \leq 1, \mu_{i}, \sigma_{i}$ are the mean and standard deviation of the $i$ th type, respectively.

(2) Interval type-2 fuzzy model

In order to improve the representation of generic uncertainty by type-1 fuzzy membership function and increase the accuracy of classification decision, fuzzing the mean and standard deviation in the type-1 fuzzy model, discreting the value in the interval $[\mu-3 \sigma, \mu+3 \sigma]$ (the confidence of real membership function in the interval $[\mu-3 \sigma, \mu+3 \sigma]$ is $99.7 \%$ ) is conducted within the interval $[\mu-3 \sigma, \mu+3 \sigma]$, and assume that the probability of occurrence of the various membership functions in the interval is the same. The interval type-2 fuzzy model with uncertain mean and standard deviation is built according to the following formula,

$$
\begin{gathered}
\mu_{i}^{-}=\mu_{i}-c_{i} \times \sigma_{i} \quad \mu_{i}^{+}=\mu_{i}+c_{i} \times \sigma_{i} \quad c_{i} \in[0,3] \\
\sigma_{i}^{-}=\frac{\sigma_{i}}{d_{i}} \quad \sigma_{j}^{+}=\sigma_{i} \times d_{i} \quad d_{i} \in[0.3,1]
\end{gathered}
$$

where $c_{i}$ and $d_{i}$ are the regulatory factors of the $i$ th membership function parameters; $\mu_{i}{ }^{-}$and $\mu_{i}{ }^{+}$as well as $\sigma_{i}{ }^{-}$ and $\sigma_{i}^{+}$respectively represent the lower bounder and upper bounder of the mean and standard deviation of the $i$ th membership function. The interval type- 2 fuzzy model is as follows:

$$
u_{j i}^{\prime}=\beta_{i} \times \exp \left\{-\frac{\left(z_{j}-\mu_{i 1}\right)^{2}}{2 \sigma_{i 1}^{2}}\right\}
$$

$u_{j i}^{\prime} \in\left[u_{j i}^{-}, u_{j i}^{+}\right], \quad \mu_{i 1} \in\left[\mu_{i}^{-}, \mu_{i}^{+}\right], \quad \sigma_{i 1} \in\left[\sigma_{i}^{-}, \sigma_{i}^{+}\right], u_{j i}{ }^{+}$and $u_{j i}{ }^{-}$ respectively stand for the upper membership and lower membership of the $j$ th to the $i$ th type, and the standard deviation remains unchanged, that is, $\sigma_{i 1}=\sigma_{i,}$ if $\mu_{i 1}=\mu_{i}^{-}, u_{j i}^{\prime}=$ ${u_{j i}^{\prime}}^{\prime}$; if $\mu_{i 1}=\mu_{i}^{+}, u_{j i}^{\prime}={u_{j i}^{\prime}}^{+}$; when the mean remains the same, that is, if $\sigma_{i 1}=\sigma_{i}^{-}, u_{j i}^{\prime}=u_{j i}{ }^{+}$; if $\sigma_{i 1}=\sigma_{i}^{+}, u_{j i}^{\prime}=u_{j i}{ }^{-}$.

The upper membership and lower membership of the interval type-2 fuzzy model with uncertain mean are calculated as follows,

$$
u_{j i}^{+}=\left\{\begin{array}{cc}
u_{j i}^{\prime-} & z_{j}<\mu_{i}^{-} \\
\beta_{i} & \mu_{i}^{-} \leq z_{j} \leq \mu_{i}^{+} \\
u_{j i}^{\prime+} & z_{j}>\mu_{i}^{+}
\end{array}\right.
$$

The upper membership and lower membership of the interval type-2 fuzzy model with

$$
u_{j i}^{-}=\left\{\begin{array}{cc}
u_{j i}^{\prime-} & z_{j} \leq \frac{\mu_{i}^{-}+\mu_{i}^{+}}{2} \\
u_{j i}^{\prime+} & z_{j}>\frac{\mu_{i}^{-}+\mu_{i}^{+}}{2}
\end{array}\right.
$$

uncertain standard deviation are calculated as follows:

$$
\begin{aligned}
& u_{j i}^{+}=\beta_{i} \times \exp \left\{-\frac{\left(z_{j}-\mu_{i}\right)^{2}}{2 \sigma_{i}^{-2}}\right\} \\
& u_{j i}^{-}=\beta_{i} \times \exp \left\{-\frac{\left(z_{j}-\mu_{i}\right)^{2}}{2 \sigma_{i}^{+2}}\right\}
\end{aligned}
$$




\subsection{Segmentation decision-making model}

The three-tier fuzzy liner neural network model is built with all the membership information of the original sampling set in the gray measure space (including the original membership, upper membership and lower membership). This neural network model adopts the supervised learning method to realize the determination of network parameter. The input vector is the set of the gray level $g(g=0,1 \ldots, 255)$ within the gray level range, and the expected output is the set of frequency values of the gray levels within the gray level range $\mathrm{y}=\left\{\mathrm{y}_{g i}, g=0,1, . ., 255, i=1, . ., m\right\}$; the number of hidden-layer nodes is three times of the number of types; the output-layer nodes is the same with the number of types. The output function adopts the piecewise linear function, as shown below(Fig 1).

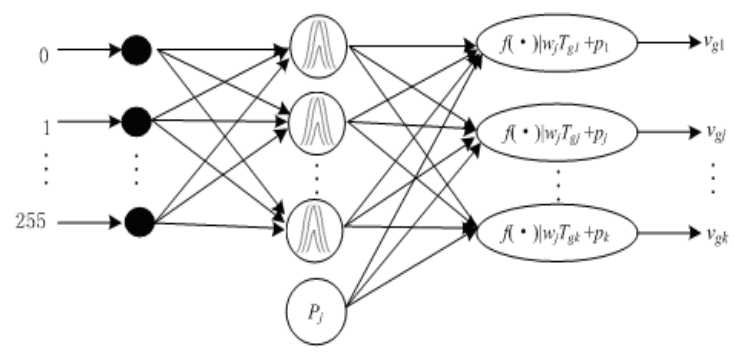

Fig 1 Fuzzy linear neural network model

The membership of the gray levels in all types of UMF and LMF as well as the membership in the original membership function (membership function in homogeneous region) are calculated and the feature vector is formed as follows:

$$
T_{g}^{\prime}=\left(U_{g 1}^{-}, U_{g 1}, U_{g 1}^{+}, \ldots, U_{g m}^{-}, U_{g m}, U_{g m}^{+}\right) \quad g=0,1, \ldots, 255
$$

where $T_{g}{ }^{\prime}$ denotes the $3 m$ dimension feature vector composed of the membership of gray level $g$ in all types. The above feature vector of all pixels in the gray measure range is taken as the out put. Then the following fuzzy linear neural network model

$$
v_{g i}=f\left(w_{i} T_{g}^{\prime}+p_{i}\right)
$$

where $0 \leq v_{g i} \leq 1$ denotes the membership of gray $g$ belongs to the $i$ th type, it satisfies the grayg belongs to the $i$ th type, it satisfies the constraint condition that

$\sum_{g=0}^{255} v_{g i}=1 . i=1, \ldots, m$ is the type index, $w_{i}=\left[w_{1 i}{ }^{+} ; w_{1 i} ; w_{1 i}\right.$ $\left.; \ldots ; w_{m i}{ }^{+} ; w_{m i} ; w_{m i}{ }^{-} ; w_{m i}{ }^{+}\right]$is the weight vector of the input neuron nodes of the $i$ th type, $p_{i}$ stands for the offset, $f$ is the activation function of output notes, which is piecewise linear function and meet the following conditions,

$$
v_{g i}=\left\{\begin{array}{lc}
w_{i} T_{g}^{\prime}+p_{i} & 1 \geq v_{g i} \geq 0 \\
0 & v_{g i}<0 \\
\max \left(y_{i}\right) & v_{g i}>1
\end{array}\right.
$$

where max $\left(y_{i}\right)$ is the maximum histogram frequency value in the $i$ th type. This paper takes the histogram frequency values of the different types of training data as the expected output, and $v_{g i}$ is the actual output. The above neural network model is built by estimating various types of weights and offsets with the least square method, and the membership matrix obtained is as follows,

$$
V=\left[v_{j i}\right]_{n \times m}
$$

In the gray space, the type of any pixel not only has relationship with the upper membership and lower membership of the type-1 fuzzy membership and type-2 fuzzy membership function, but also with the membership of the neighborhood pixels to the $i$ th type. The larger the membership of the neighborhood pixels belong to the $i$ th type is, the higher membership of the $j$ th pixel belongs to the $i$ th type will be. The segmentation decision-making model integrated with spatial relationship is built based on the above principle,

$$
f_{j i}=\frac{1}{\# N_{j}} \sum_{k \in N_{j}} v_{k i}
$$

where $f_{j i}$ represents the segmentation decision-making membership of the $j$ th pixel to the $i$ th type, $N_{j}$ is the pixel membership set in the $3 \times 3$ window with the pixel $j$ as the center, $\# N_{i}=9$ is the number of the pixels in the window, $k=1$, $2, \ldots, 9$ is the index of neighborhood pixels.

The segmentation decision-making membership matrix is as follows:

$$
U^{*}=\left[f_{j i}\right]_{n \times m}
$$

In order to obtain a clear segmentation result, the max membership principle is used to realize the regional division of the image,

$$
M_{j}=\arg _{i}\left\{\max \left\{f_{j i}\right\}\right\} \quad j=1, \ldots, n ; \quad i=1, \ldots, m
$$

Where $M_{j}$ stands for the type of the jth pixel, and $\boldsymbol{M}=$ $\left\{M_{1}, M_{2}, \ldots, M_{\mathrm{n}}\right\}$ denotes the clear regional result.

\section{EXPERIMENTAL RESULTS AND DISCUSSION}

To illustrate the feasibility and effectiveness, segmentation experiments are respectively tested on the synthetic high-resolution remote sensing images and the real high-resolution remote sensing images, and the quantitative and qualitative evaluations are made.

\subsection{Synthetic high-resolution remote sensing image}

Fig. 2 shows four ground objects clipped from the real World View-II panchromatic image. The resolution is $0.5 \mathrm{~m}$, and the pixel size is $256 \times 256$. I - IV region respectively represent cropland, partially frozen water area, concrete pavement, and forest.

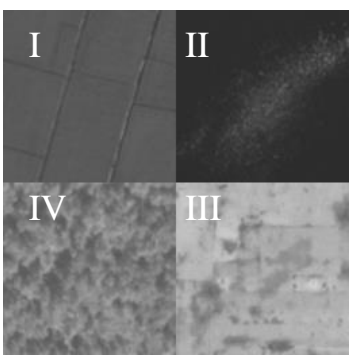

Fig 2 World view-2 synthetic images

Fig. 3 (a) is the type-1 fuzzy model obtained by supervised sampling to each region and the histogram matching with the least square method, namely, Gaussian function model ; Fig. 3 (b) shows the best fuzzy segmentation model obtained by applying the method proposed in this paper and without considering the neighborhood relationship. The four curves with different colors stand for the histogram matching models of the four kinds of ground objects,and the symbols "**, "++", " $乞$ " and " $\triangle$ " respectively denote the training sample frequency values of the four regions.

Fig 4 shows the segmentation results of synthetic images 
obtained by different methods: In Fig. (a), the segmentation results of type-2 fuzzy neuron networks without considering the spatial relationship; In Fig. 4 (b) the standard FCM segmentation result; In Fig. 4 (c) the FCM segmentation result of Hidden Markov Random Field (HMRF) (HMRF-FCM); In Fig. 4 (d) the segmentation result by the method integrated with spatial relationship proposed in this paper.

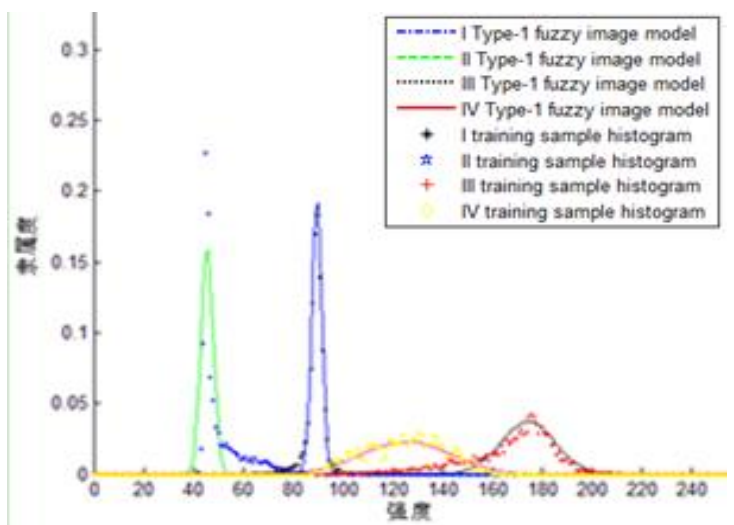

(a) Type-1 fuzzy model
In order to evaluate the accuracy of the above different segmentation results, the confusion matrixes with the segmentation results in Fig. 3 (a)-(d) are obtained based on the standard templates shown in Fig. 1, and the product precision, user precision, overall precision and kappa coefficient are respectively calculated and listed in Table 1

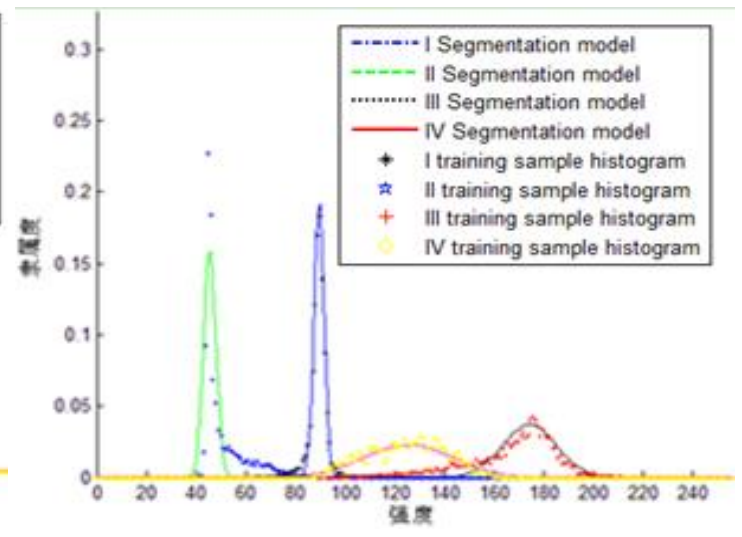

(b) Best fuzzy segmentation model

Fig 3 Fuzzy model

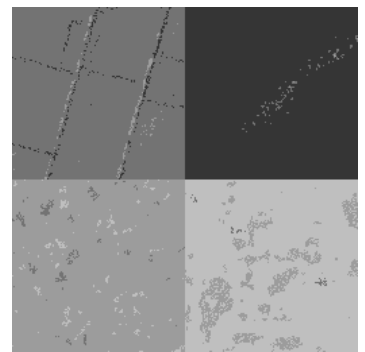

(a) Segmentation of type-2 fuzzy neuron networks

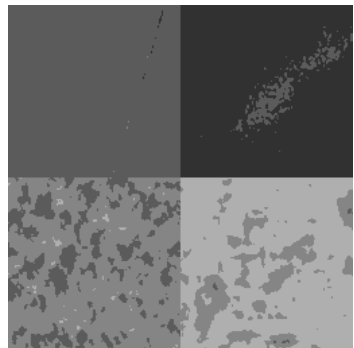

(b) Segmentation of FCM

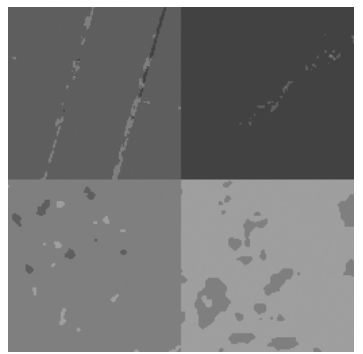

(c) Segmentation of HMRF-FCM

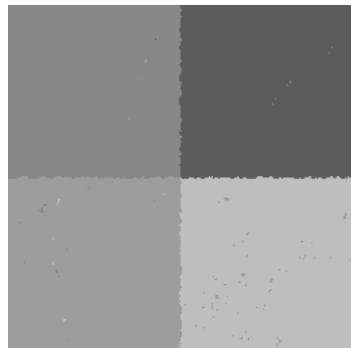

(d) Segmentation of proposed algorithm

Fig.4 Segmentation of synthetic

\begin{tabular}{|c|c|c|c|c|c|}
\hline \multirow{2}{*}{ Methods } & \multirow{2}{*}{ Precision indexes } & \multicolumn{4}{|c|}{ Homogeneous regions } \\
\hline & & I & II & III & IV \\
\hline \multirow{3}{*}{$\begin{array}{l}\text { Interval type-2 fuzzy } \\
\text { neural network }\end{array}$} & User precision & 0.972 & 0.992 & 0.980 & 0.884 \\
\hline & Product precision & 0.979 & 0.990 & 0.884 & 0.966 \\
\hline & \multicolumn{5}{|c|}{ Overall precision $=0.955 ; \mathrm{kappa}=0.940$} \\
\hline \multirow{4}{*}{ FCM } & User precision & 0.770 & 0.999 & 0.986 & 0.789 \\
\hline & Product precision & 0.998 & 0.945 & 0.799 & 0.747 \\
\hline & \multicolumn{5}{|c|}{ Overall precision $=0.872 ; \mathrm{kappa}=0.830$} \\
\hline & User precision & 0.977 & 0.993 & 0.986 & 0.871 \\
\hline \multirow[t]{2}{*}{ HMRF-FCM } & Product precision & 0.966 & 0.992 & 0.882 & 0.972 \\
\hline & \multicolumn{4}{|c|}{ Overall precision $=0.953 ;$ kappa $=0.937$} & \\
\hline \multirow{3}{*}{$\begin{array}{l}\text { Algorithms in this } \\
\text { paper }\end{array}$} & User precision & 0.994 & 0.995 & 0.997 & 0.991 \\
\hline & Product precision & 0.997 & 0.997 & 0.990 & 0.994 \\
\hline & \multicolumn{4}{|c|}{ Overall precision $=0.994 ; \mathrm{kappa}=0.992$} & \\
\hline
\end{tabular}

Table 1 Comparison of precision and kappa value

(1) In Fig. 2, in order to reflect the detail features of high-resolution remote sensing image and the complexity of the distribution features of the homogeneous regions, the synthetic high-resolution remote sensing image, involving four typical ground objects: the partially frozen water area, the concrete 
pavement with different shades, the forest with big gray feature difference, and cropland, is tested using the proposed approach. The frequency value distribution of the training samples in different regions (shown in Fig. 2) shows that, the distribution features of the other three ground objects are all complex, except that the cropland conforms to the Gaussian distribution feature. The fitting effects of type-1 fuzzy image model on the four different regions (Fig 2. (a)) are bad except for the cropland; but the proposed method can highly fit the above four kinds of ground objects (Fig. 2 (b)).

(2) In Fig 4, because the FCM algorithm can only deal with medium-low resolution images and is sensitive to noise, the cropland segmentation result (Fig. 4 (b)) of FCM algorithm is better than the one of HMFR-FCM (Fig. 4 (c)), large amount of pixels of the IV ground object Forest are misclassified into the cropland, and there are many misclassified pixels existing in the III ground object due to large color difference. Compared with the other three methods, the segmentation accuracy of the standard FCM method is the lowest; HMRF-FCM algorithm (Fig. 4 (c)) takes the correlation of neighborhood pixel into consideration, so it can overcome the noise effect and the segmentation result of the four ground objects are better than the standard FCM algorithm result. But it doesn't take the segmentation decision-making influence into consideration and cannot deal with the clustered noise, such as the dark area of the cement road. Thus, its segmentation result is slightly lower than that of interval type-2 fuzzy neural network (Fig. 4 (a)); Fig 3 (a) shows that the segmentation model based on the interval type-2 fuzzy neural network can match the complex training data histogram distribution characteristics of high-resolution remote sensing image to greatly improve the segmentation accuracy. But it is sensitive to noise and there is large number of salt and pepper noise in the segmentation result; to overcome of disadvantages of the segmentation model based on interval type-2 fuzzy neural network, the proposed method has integrated the spatial relationship into the membership spatial domain, effectively solving the above problems. Therefore, its segmentation result is the best with a little sparse noise.

(3) Table 1 lists that the segmentation model based on interval type-2 fuzzy neural network adopts the supervised sampling. The segmentation model in this paper can match the training data histogram in high accuracy, with its overall precision 0.955, and kappa value 0.940. But the precision of the HMRF-FCM segmentation method (overall precision is 0.953 and the kappa value is 0.937) is slightly lower than that of the method based on interval type- 2 fuzzy neural network; for the segmentation of the above four ground objects, the lowest segmentation precision of the standard FCM method is 0.789 , the method based on interval type-2 fuzzy neural network is 0.884 , and the HMRF-FCM is 0.871; while the lowest precision of the method proposed in this paper is 0.99 , and both its overall precision and kappa value have been more than 0.99, which has realized high-precision segmentation of high-resolution synthetic image.

\subsection{Real high-resolution remote sensing image}

Fig. 5 (a) shows a World View-2 panchromatic image with the resolution of $0.5 \mathrm{~m}$. The colors from light to heavy respectively represents the house, road and lawn in order; Fig. 5 (b) and (c) show the $0.6 \mathrm{~m}$ resolution QuickBird panchromatic images. In Fig. (b), the colors from light to heavy respectively stands for road, forest and water area in order. Fig. 5 (c) shows the limestone, granite and water area, and their image sizes are all $256 \times 256$.

Fig. 6 (a1)-(c1) show the segmentation results of the three images (in Fig. 6) obtained by applying the segmentation method based on the interval type-2 fuzzy neural network; Fig.6 (a2)-(c2) are the segmentation results obtained by standard FCM; Fig. (a3)-(c3) are the segmentation results by HMRF-FCM; Fig. (a4)-(c4) are the segmentation results obtained by the proposed method. For the segmentation results shown in Fig. 5 (a), compared with other three methods, although HMRF-FCM can greatly reduce the noise of the three ground objects, it misclassifies partial adjacent roads into the house, and the light-colored part of the road is also misclassified into house. That is, it has severe over-segmentation, which lowers the overall segmentation precision; the segmentation of road, HMRF-FCM is the worst, and the interval type-2 fuzzy neural network is close to the standard FCM; for the segmentation of lawn, HMRF-FCM is the best, and standard FCM is slightly better than the segmentation method of (a1). For the segmentation results of the three ground objects, the proposed method is the best compared with the above three segmentation methods. For the segmentation of forest in Fig. 5 (b), the proposed method is still the best, which only has a little noise; the interval type-2 fuzzy neural network is close to the HMRF-FCM; the FCM is the worst; for the segmentation of road, HMRF-FCM is close to the method proposed in this paper, both of which are better than the standard FCM algorithm; due to the smooth of the gray features of water area, the segmentation results by the above four methods are almost the same. In Fig 5. (c), the overlapping range of histogram distribution characteristics between the limestone and water area is large. If the segmentation difficulty in the neighborhood relationship is not taken into consideration, the segmentation result of the method based on interval type-2 fuzzy neural network is the worst compared with the other three methods; for 
the segmentation of limestone and granite, the method proposed in this paper is the best, HMRF-FCM is better than the method based on interval type-2 fuzzy neural network, and the standard FCM is the worst. The segmentation

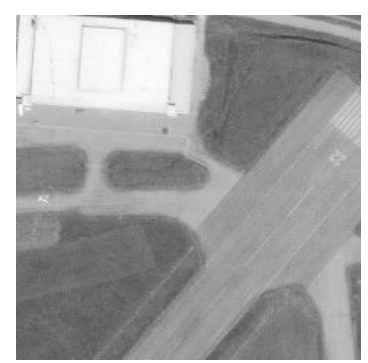

(a) World View-II image

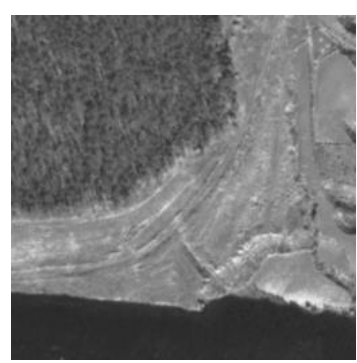

(b) QuickBird image

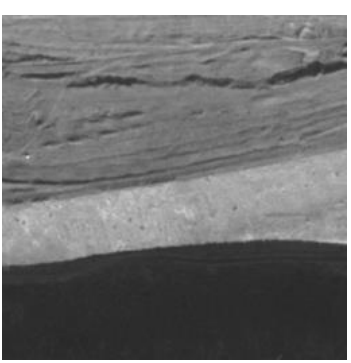

(c) QuickBird image

Fig 5 High resolution remote sensing image

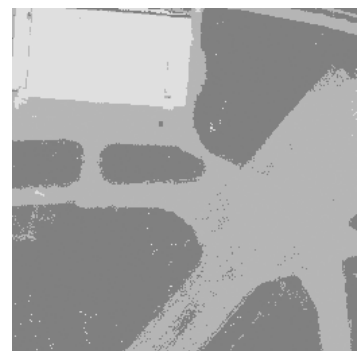

(a1) Segmentation of type-2 fuzzy neuron networks

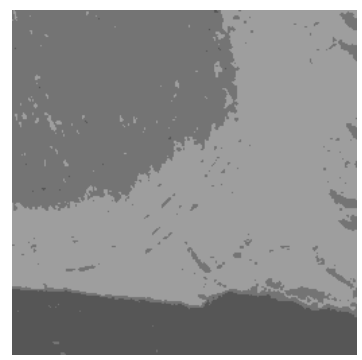

(b1) Segmentation of type-2 fuzzy neuron networks

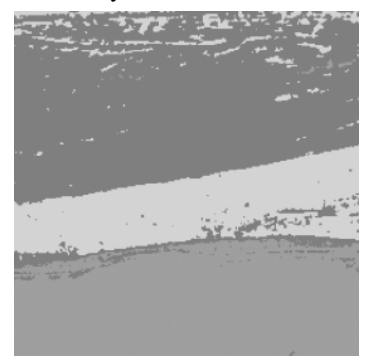

(c1) Segmentation of type-2 fuzzy neuron network

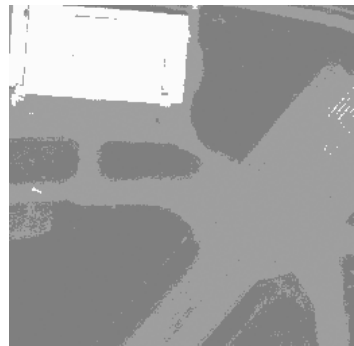

(a2) Segmentation of FCM

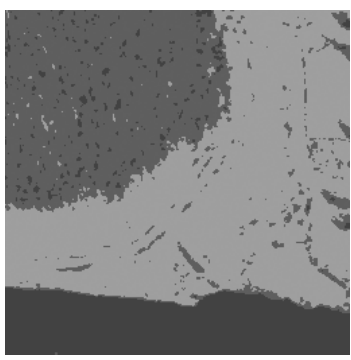

(b2) Segmentation of FCM

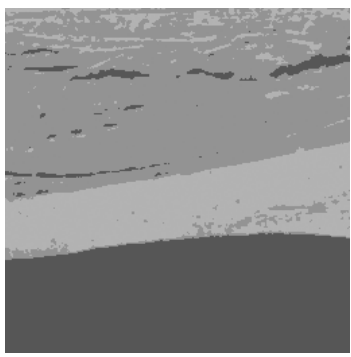

(c2) Segmentation of FCM

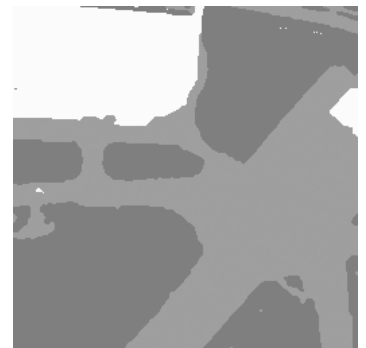

(a3) Segmentation of HMRF-FCM

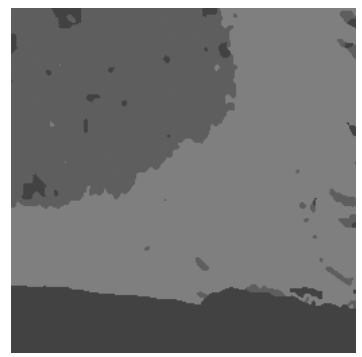

(b3) Segmentation of HMRF-FCM

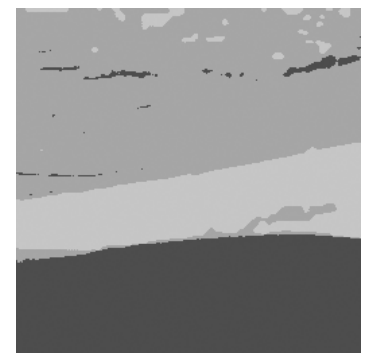

(c3) Segmentation of HMRF-FCM

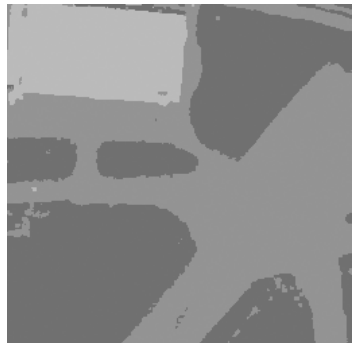

(a4) Segmentation of proposed algorithm

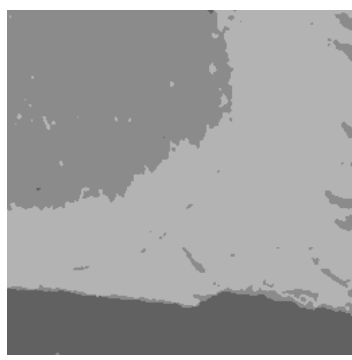

(b4) Segmentation of proposed algorithm

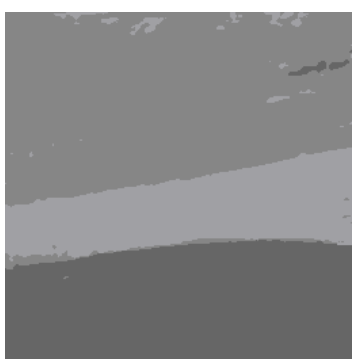

(c4) Segmentation of proposed algorithm

Fig.6 Segmentation of image

\section{CONCLUSION}

In this paper, puts forward a supervised segmentation method of high-resolution remote sensing image based on the interval type-2 fuzzy neural network is proposed. This method can deals with the pixel generic uncertainty by building the type-1 fuzzy image model; and the interval type-2 fuzzy model is built to describes the uncertainty of segmentation decision-making by building the interval type-2 fuzzy model; then take s the primary membership as well as the upper and lower membership of each pixel by supervised sampling in all types as the input to build the fuzzy linear neural network model; and finally 
realizes fuzzy classification by building neighborhood relationship in the membership domain is built to realize fuzzy classification. In order to verify the feasibility and validity of the algorithm proposed algorithm min this paper, the interval type-2 fuzzy neural network method which does not integrate the spatial relationship, the standard FCM algorithm, HMRF-FCM which integrates spatial relationship, and the proposed method proposed in this paper are respectively applied to tested on conduct the segmentation tests on the of high-resolution synthetic images and real images. The quantitative and qualitative analysis results verifyies that the proposed method proposed in this paper can improve the segmentation precision. Although this method can restrain the noise impact in the area and realize better segmentation to the high-resolution remote sensing image, it has the fuzzy boundary phenomenon. Therefore, the researches are focus on the above problems to further improve the algorithm in the further.

\section{ACKNOWLEDGMENTS}

This work was supported by Research Fund for the Doctoral Program of Higher Education of China (No. 20122121110007) and National Natural Science Foundation of China (No. 41271435; No. 41301479).

\section{REFERENCE}

Ahmed, M. Yamany, S. Mohamed, N. Faragand, A. Moriarty, T., 2002. A modified fuzzy c-means algorithm for bias field estimation and segmentation of MRI data. IEEE Transactions On Medical Imaging, 21(3), pp. 193-199.

Ahmadieh, K. M. Erdal, K. Mojmmad, T. et al., 2011. Analysis of the noise reduction property of type-2 fuzzy logic systems using a novel type-2 membership function. IEEE Transactions on Systems Man and Cybernetics Part B Cybernetics, 41(5), pp. 1395-1406.

Bezdek, J., 1981. Pattern recognition with fuzzy objective function algorithms. New York: Plenum, pp. 79-88.

Bruzzone, L. and Carlin, L., 2006. A multilevel context-based system for classification of very high spatial resolution images. IEEE Transactions on Geoscience and Remote Sensing, 44(9), pp. 2587-2600.

Castillo, O. Melin, P., 2004. Adaptive noise cancellation using type-2 fuzzy logic and neural networks. Proceedings of the 2004 IEEE International Conference on Fuzzy Systems, 2(7), pp. 1093-1098.

Cai, W. Chen, S. Zhang, D., 2007. Fast and robust fuzzy c-means clustering algorithms incorporating local information for image segmentation. Pattern Recognition, 40(3), pp. 825-838.

Chatzisand, S. P. and Varvarigon, T. A., 2008. A fuzzy clustering approach toward hidden Markov random field models for enhanced spa1109tially constrained image segmentation. IEEE Transactions on Fuzzy System, 16(5), pp. 1351-1361.

Jonr, I. Innocent, P. R. Bames, M. R., 2000. Neuro-fuzzy clustering of radiographic tibia image data using type-2 Fuzzy Sets. Information Sciences, 125(1-4), pp. 65-82.

Juang, C. F. Tsao, Y. W., 2008 A type-2 self-organizing neural fuzzy system and its FPGA implementation. IEEE Transactions on Systems Man and Cybernetics Part B Cybernetics A Publication of the IEEE Systems Man and Cybernetics Society, 38(6), pp. 1537-48.

Karnik, N. N. Mendel, J. M. Liang Q., 1999. Type-2 fuzzy logic systems [J]. IEEE Transactions on Fuzzy Systems, 7(6), pp. 643-658.

Karnik, N. N. Mendel, J. M., 2001. Centroid of a type-2 fuzzy Set. Information Sciences, 132(1), pp. 195-220.

Liang, Q. Mendel, J. M., 2001. Mpeg VBR video traffic modeling and classification using fuzzy technique [J]. IEEE Trans on Fuzzy Systems, 9(1), pp. 183-193.

Liu, S. Y. Li, X. F. Li, H. G. Li, Z. M., 2007. Research of fast image segmentation based on Markov spatial constrain and fuzzy c-means clustering. Application Research of Computers, 24(8), pp. 220-223.

Lin, Y. Y. Liao, S. H. Lin, C. Y., 2014. Simplified interval type-2 fuzzy neural networks. IEEE Transactions on Neural Networks and Learning Systems, 25(5), pp.959-969.

Mendel, J. M., 2000. Uncertain rule-based fuzzy logic systems: Introduction and New Directions. Prentice Hall PTR.

Melin, P. Solano, D. Soto, M. et al., 2006. Voice recognition with neural networks, type-2 fuzzy logic and genetic algorithms. Journal of Engineering Letters, 13(2), pp. 08-116.

Sanchez, D. Melin, P., 2010. Modular neural network with fuzzy integration and its optimization using genetic algorithms for human recognition based on iris, ear and voice biometrics. Studies in Computational Intelligence, 312(2), pp. 127-144. 